1 Universidade de

Pernambuco (UPE) e

Universidade Estadual da Paraíba (UEPB),

Programa Associado de Pós-Graduação em Enfermagem - Recife (PE), Brasil.

rebecaangelim@hotmail.com

2 Universidade de Pernambuco (UPE) e

Universidade Estadual da Paraíba (UEPB),

Programa Associado de Pós-Graduação em

Enfermagem - Recife (PE),

Brasil.

vmirelle@gmail.com

3 Universidade de Pernambuco (UPE) e Universidade Estadual da Paraíba (UEPB),

Programa Associado de Pós-Graduação em Enfermagem - Recife (PE), Brasil.

daniela_3439@hotmail.com

4 Universidade de Pernambuco (UPE) e Universidade Estadual da Paraíba (UEPB), Programa Associado de Pós-Graduação em Enfermagem - Recife (PE), Brasil.

bri.melo@hotmail.com

5 Universidade de Pernambuco (UPE) e Universidade Estadual da Paraíba (UEPB),

Programa Associado de Pós-Graduação em Enfermagem - Recife (PE), Brasil.

fatimaabrao@hotmail.com

\section{Representações sociais de estudantes de escolas públicas sobre as pessoas que vivem com HIV/Aids}

\author{
Social representations of public school students about people living \\ with HIV/Aids
}

Rebeca Coelho de Moura Angelim1, Verônica Mirelle Alves Oliveira Pereira², Daniela de Aquino Freire $^{\mathbf{3}}$, Brígida Maria Gonçalves de Melo Brandão4, Fátima Maria da Silva Abrão5
RESUMO O estudo objetivou analisar as representações sociais dos jovens e adultos acerca de suas vivências ante a pessoa com Vírus da Imunodeficiência Humana/Síndrome de Imunodeficiência Adquirida (HIV/Aids). Trata-se de um estudo descritivo, de caráter exploratório com abordagem qualitativa, desenvolvido com 59 alunos do Programa de Educação de Jovens e Adultos, matriculados em duas escolas do município de Recife. Utilizou-se a técnica de entrevistas. Os dados foram analisados com apoio do software Alceste 4.5. Conclui-se que há necessidade de integrar temas sobre a conscientização social acerca da Aids. Ressalta-se a importância do enfermeiro como facilitador em colaborar para a desmistificação de sentimentos e atitudes referentes às pessoas com HIV.

PALAVRAS-CHAVE Síndrome de Imunodeficiência Adquirida. HIV. Adulto jovem. Percepção.

ABSTRACT The study aimed to analyze the social representations of young people and adults about their experiences facing the person with HIV/AIDS. It is a descriptive, exploratory study with qualitative approach, developed with 59 students of the Youth and Adult Education Program, enrolled in two schools in the city of Recife. We use the technique of interviews. Data were analyzed with the support of software Alceste 4.5. It is concluded that there is a need to integrate issues of social awareness about AIDS. We emphasize the importance of the nurse as a facilitator in helping to demystify feelings and attitudes towards people with HIV.

KEYWORDS Acquired Immunodeficiency Syndrome. HIV. Young adult. Perception. 


\section{Introdução}

O Vírus da Imunodeficiência Humana (HIV) e a Síndrome da Imunodeficiência Adquirida (Aids) são temas discutidos mundialmente (DANTAS ET AL., 2015). Desde o início da década de 1980, a identificação do HIV/ Aids constitui um desafio para a comunidade científica global, sendo considerado um problema de saúde pública, de grande magnitude e caráter pandêmico que envolve diversos atores sociais, atingindo os indivíduos sem distinção social, econômica, racial, cultural ou política (PERUCCHI ET AL., 2011).

No Brasil, o primeiro caso foi registrado no estado de São Paulo na década de 1980. Comparando a evolução da epidemia de Aids com dados disponíveis desde esse período, chama atenção o fato de que, pela primeira vez em sete anos, a taxa de detecção por 100 mil habitantes caiu para menos de 20 casos, passando a ser a menor taxa de detecção dos últimos 12 anos (PERUCCHI ET AL., 2011). A maior concentração dos casos de Aids no Brasil está nos indivíduos com idade entre 25 e 39 anos para ambos os sexos, porém, no que se refere às faixas etárias, observa-se que a maioria dos casos de infecção pelo HIV encontra-se nas faixas etárias de 15 a 39 anos (BRASIL, 2015).

A abordagem sobre o HIV/Aids pode ser realizada por meio de programas em saúde que envolvem os estudantes tanto individualmente quanto em grupo, usando como base palestras e/ou dinâmicas diversas acerca das Doenças Sexualmente Transmissíveis (DST), inclusive a Aids (NAgAMATSU ET AL., 2011). Além disso, alguns fatores podem influenciar no melhor entendimento dos alunos sobre essas doenças, como a participação da família, qualidade de vida e a educação (CAI ET AL., 2012).

Desse modo, torna-se imprescindível a realização de práticas educativas voltadas à temática da sexualidade, principalmente no ambiente escolar a respeito da prevenção do HIV, visto que a escola atua como agente facilitador no processo de aprendizagem dos estudantes, estabelecendo uma troca de informação dos alunos com os educadores (PIAU; PEREIRA, 2012).

Vale ressaltar que em relação à fonte de informações dos adolescentes sobre DST e o HIV/Aids, 60,6\% afirmaram utilizar a televisão como principal meio para obter informações acerca dessas doenças, seguido por $49,3 \%$ que referiram a escola com o professor como principal fonte disseminadora de informação (CHAVES ET AL., 2014).

Dessa forma, salienta-se a importância da escola como um ambiente onde se trabalha conhecimentos, habilidades e mudanças comportamentais em diferentes áreas do conhecimento humano, tornando-se o local mais adequado para as ações educativas e promotoras em saúde (CAMARGO; FERRARI, 2009). Além de ser um ambiente ideal no processo ensino-aprendizagem, a escola é fundamental na formação de pessoas no que tange aos direitos civis e de proteção à pessoa física, promove a inserção nos diversos aspectos sociais e de políticas públicas e, com as demais entidades promotoras do desenvolvimento de jovens e adultos, propicia um local indispensável para a abordagem acerca da temática HIV/Aids (BRASIL, 2009).

Estudos que promovam a reflexão acerca do impacto do HIV/Aids na sociedade e a atuação do profissional de saúde como facilitador de diálogo, das questões suscitadas pela doença, reforçam a necessidade de estudar as representações sociais sobre 0 processo do adoecer pelo vírus.

Segundo Denize Jodelet (2001), a falta de informações e incertezas científicas promove o surgimento de pensamentos, ou seja, das representações sociais que circulam em torno de uma comunidade. Essas definições compartilhadas pelos indivíduos de um mesmo grupo suscitam em uma realidade para eles, que pode ou não entrar em conflito com outros grupos. Nessa perspectiva, a autora afirma que as representações são um sistema de interpretação que promove a relação do indivíduo com o mundo, podendo representar ou se representar a um objeto ou a um sujeito. 
Diante do exposto, trabalhar representações sociais em um ambiente escolar, tendo em vista a análise do conhecimento social compartilhado sobre um determinado tema, na visão do senso comum dessa população em um ambiente educacional é de fundamental importância.

Nesse caminhar, a problemática deste estudo partiu de que o conhecimento acerca do HIV/Aids entre estudantes de escola pública ainda é deficiente em face de ser um grupo vulnerável com reduzido acesso as políticas públicas de promoção da saúde.

O pressuposto teórico se deu pelo fato de se tratar de uma população que apresenta elevado número de contaminação pelo HIV/ Aids e que pode dispor de informações pertinentes sobre a doença para a equipe de saúde no que se refere às representações sociais. Dessa forma, este estudo objetivou analisar as representações sociais dos jovens e adultos acerca de suas vivências perante pessoas que vivem com HIV.

\section{Métodos}

Trata-se de um estudo descritivo, de caráter exploratório com abordagem qualitativa. A pesquisa foi desenvolvida em duas escolas estaduais que possuem o curso presencial de Educação de Jovens e Adultos (EJA), foi realizada no período de agosto a dezembro 2013, na cidade do Recife, Brasil. O critério para os estudantes participarem da pesquisa foi o de estarem regularmente matriculados nas escolas contempladas e inseridos na modalidade de ensino EJA.

Os dados foram coletados a partir da aplicação de um questionário aos 169 alunos, com idade entre 18 e 63 anos, o qual objetivou identificar as possíveis pessoas contaminadas pelo HIV ou sujeitos que conhecem pessoas infectadas; assim, somente os sujeitos que têm esse conhecimento ou são contaminados seguiram para a entrevista. Desse modo, 59 alunos afirmaram conhecer pessoas com o HIV. Nenhum aluno afirmou possuir o vírus. As entrevistas obedeceram um roteiro previamente elaborado com sete questões, sendo que, para análise deste estudo, a ênfase foi dada para a seguinte questão: Como você percebe/visualiza uma pessoa contaminada com HIV/Aids? Os indivíduos foram entrevistados individualmente. Cada entrevista durou em média 15 minutos, o conteúdo foi gravado em aparelho MP3, com posterior transcrição.

Os dados qualitativos foram dispostos no programa Alceste - Análise Lexical por Contexto de um Conjunto de Segmentos de Texto. As entrevistas foram transcritas em um documento Word ${ }^{\circledR}$, em que foram minuciosamente revisadas, sendo eliminados os vícios de linguagem e adequada a ortografia. Posteriormente, foram operacionalizadas conforme o programa, por meio de um corpus de natureza textual, de análise standard, ou seja, a utilização de entrevistas com questões abertas (OLIVEIRA; GOMES; MARQUES, 2005).

A montagem do corpus se deu por intermédio da apresentação das variáveis chamadas de linhas estreladas, que delimitam cada unidade a ser analisada. As variáveis usadas foram sexo e escola, as quais foram utilizadas apenas a critério de identificação do sujeito. Criou-se um dicionário para que houvesse uma redução de vocabulário; as palavras com o mesmo significado foram substituídas por um único termo com o intuito de homogeneizar o texto.

De acordo com as regras do software, também foram substituídas palavras que tivessem o hífen para o underline, e as falas do entrevistador foram colocadas em letras maiúsculas para que não se confundissem com a fala do entrevistado (NASCIMENTO; MENANDRO, 2006). As 59 entrevistas realizadas totalizaram 47 páginas com 67.094 caracteres, o corpus foi inspecionado por uma das pesquisadoras especialista nesse tipo de análise durante toda a sua montagem.

Após a leitura dos conteúdos posteriores a cada linha estrelada ou da Unidade de Contexto Inicial (UCI), comando que o 
programa identifica onde se inicia cada entrevista, o Alceste segmenta o texto em Unidades de Contexto Elementar (UCE), que são fragmentos menores apresentados em três ou mais linhas (NASCIMENTO; MENANDRO, 2006).

Por fim, são criadas as classes, que são conjuntos de UCE com sentidos semelhantes e vocabulários homogêneos. A análise dos resultados apresentados pelo Alceste foi ancorada em autores que abordam as representações sociais.

Para o desenvolvimento desta pesquisa, foi respeitada a norma e diretriz para a realização de pesquisas envolvendo seres humanos, contidas na Resolução $\mathrm{n}^{\circ}$ 446/2012, do Conselho Nacional de Saúde (CNS). A pesquisa foi aprovada pelo comitê de Ética da Universidade de Pernambuco com parecer datado no dia 2 de julho de 2013 de $n^{0} 323.058$.

\section{Resultados e discussão}

A análise do resultado do software Alceste partiu de 59 linhas estreladas, o corpus íntegro apresentou $73 \%$ de aproveitamento. De acordo com a distribuição das UCE, a classe que associou o predomínio textual com o objetivo proposto foi a classe $3 \mathrm{com}$ $43 \%$ do corpus. Para obter melhor compreensão da classe analisada, ela foi nomeada para atender ao objetivo da pesquisa como: 'O significado das representações sociais dos jovens e adultos e suas percepções acerca da pessoa com HIV'.

As principais formas reduzidas, o contexto semântico e os respectivos valores de qui-quadrado $\left(\mathrm{x}^{2}\right)$ constituintes da classe 3 que possibilitam uma compreensão melhor do conteúdo da classe são apresentadas no quadro 1.

Quadro 1. Principais formas reduzidas, contextos semânticos e valores de $x^{2}$ da classe 3

\begin{tabular}{llc}
\hline \multicolumn{1}{c}{ Formas reduzidas } & \multicolumn{1}{c}{ Contexto semântico } & KH12 \\
\hline Viv & vive(1) vivem(1) viver(12) vivia(4) vivo(1) & 15 \\
Acontec & acontece(1) acontecer(14) acontecesse(1) aconteceu(3) acontecia(1) & 14 \\
CUR & cura(12) curar(1) & 13 \\
Amigo & amigo(9) amigos(4) & 11 \\
Descuid & descuidar(1) descuido(8) descuidou(1) & 10 \\
Trist & triste(17) tristes(1) tristeza(4) & 9 \\
Compaixão & compaixão(12) & 8 \\
Difícil & difícil(9) & 8 \\
Depressão & depressão(5) & 7 \\
\hline
\end{tabular}

Na Classificação Hierárquica Ascendente (CHA), resultado apresentado pelo software, foram consideradas 21 formas reduzidas. Observa-se que há uma ligação entre: cura + convívio + compaixão + difícil, sequência que forma a primeira temática: 'Vivências e sentimentos de jovens e adultos a pessoas com HIV’. Há também uma associação entre: amigo + coisa + viver + podemos + aconteceu + acho, cuja temática denomina-se: 'Lembranças e hipóteses de como ocorreu a transmissão do HIV'. As palavras: acabou + faz + vez + penso, constituem a temática: 'Dimensões e complexidades observadas 
pelos estudantes a pessoas com HIV'. As formas: tomar + sabemos + vou + uma + triste + vida formam a temática: 'Vulnerabilidade de pessoas com o HIV devido à infecção do vírus'.

Com vista no objetivo de analisar as representações sociais dos jovens e adultos inseridos no EJA com base na classe escolhida, nas UCE analisadas e as formas reduzidas emergidas do software, surgiram os resultados correspondentes ao grupo em geral independentemente das instituições pesquisadas.

As quatro divisões binárias propostas pela CHA da classe 3 serão em seguida abordadas.

\section{Vivências e sentimentos de jovens e adultos a pessoas com HIV/Aids}

As informações contidas nessa categoria trazem as experiências vividas pelos atores da pesquisa, e os sentimentos expostos por eles nas entrevistas fazem referência à tristeza, à depressão, à compaixão e à amizade como acolhimento crucial para a retomada de laços para sociabilidade do portador. As falas remetem essa realidade:

Uma fatalidade, mesmo tendo uma vida boa, no final ele sabe o que pode acontecer, então é viver em uma corda bamba se equilibrando para não cair, mas no final de tudo ele sabe o que vai acontecer, é triste. (E 05).

Fiquei triste porque eu o conheço desde criança e independente da sexualidade, ele é ser humano, estou do lado dele. (E 53).

É necessária a compreensão emocional dos amigos, familiares e cônjuges que circundam o portador, adequando suas orientações e cuidados durante o adoecer pelo vírus. Por este motivo, faz-se necessária a presença do profissional de saúde no ambiente educacional, ajudando a promover a desmistificação de crenças e a inversão dos valores associados aos sentimentos de negatividade ligados aos portadores.
Para tanto, destaca-se o Programa Saúde na Escola, o qual vai além de um programa de governo para uma política de Estado, pois implica na superação do modelo biomédico de atenção aos estudantes para uma proposta promotora de saúde, com a participação efetiva dos gestores, profissionais das Estratégias Saúde da Família (ESF) e da escola e comunidade escolar (estudantes, pais, comunidade do entorno), com base no enfrentamento de suas necessidades específicas, proporcionando o trabalho com capacidades individuais e coletivas existentes (FERREIRA ET AL., 2014)

Os sujeitos expuseram tais depoimentos e concepções reportando-se às suas memórias sociais. A reapresentação do sofrimento torna-se real quando esses sujeitos se deparam com a realidade da Aids, uma doença incurável, o que determina sentimentos tão intensos e angustiantes centralizados na amargura, tristeza, desespero, solidão, medo da morte e da rejeição (CAMARGo; Bertolo; BÁRBARA, 2009; SERRA ET AL., 2013).

Assim, percebe-se um conjunto de representações, vivências e sentimentos que, direta ou indiretamente, influenciaram e deixaram marcas significativas nas trajetórias dos entrevistados, sobretudo em relação aos sentimentos vivenciados.

Também têm destaque para o sujeito da pesquisa o respeito e o acolhimento como pontos essenciais para pessoas com HIV. A fala seguinte expressa o respeito, a solidariedade à pessoa com o vírus:

[...] é mais fácil quando você lida diretamente com a pessoa, você aprende a respeitar, a se prevenir, a ajudar as outras pessoas, e também a entender que o nosso mundo está sujeito a acontecer qualquer coisa. (E 22).

Essas representações estão integradas a um valor social, associadas às repercussões afetivas e morais negativas, ligadas ao fato de estar doente. 


\section{Lembranças e hipóteses de como ocorreu a transmissão do HIV/Aids}

Nessa subclasse, foram identificados dois tipos de abordagens. Na primeira, os participantes visualizam as principais formas de transmissão do vírus cientificamente comprovadas, como a relação sexual desprevenida, a transmissão por meio de objetos perfurocortantes contaminados e transfusão sanguínea. Evidenciam o uso de preservativos como o meio mais eficaz para a prevenção das DST, reapresentam a relação sexual como forma principal da transmissão do HIV. A fala seguinte evidencia essa realidade.

Irresponsabilidade. Porque hoje em dia está tudo muito aberto, televisão tem uma comunicação grande, as pessoas falam uns com os outro, até as crianças de hoje sabem o que é preservativo. (E 16).

A citação do preservativo nos depoimentos é de extrema importância por se tratar de um método que, além de proteger contra DST, funciona como um contraceptivo. Destaca-se a importância educativa do profissional de saúde no ambiente educacional, que possa promover reflexões às práticas sexuais preventivas e o manuseio adequado dos métodos preventivos. O uso do preservativo apresenta-se como peça-chave para o enfrentamento da epidemia (CAMARGO; BERTOLO; BÁRBARA, 2009).

Vale destacar ainda algumas estratégias adotadas para a diminuição do risco de contaminação de HIV/Aids; dentre elas estão: o conhecer de saberes e práticas, a pesquisa comportamental, o diálogo, a humanização, a consulta de enfermagem, a capacitação profissional, o cuidado compartilhado e o planejamento de ações (SANTOS ET AL., 2012).

A segunda abordagem demonstra que os participantes julgam o descuido e a irresponsabilidade nos intercursos sexuais como principais causas de contaminação. Os depoimentos relatam os mitos sobre a transmissão do vírus, ainda presentes nas populações adjacentes aos atores da pesquisa.

As pessoas acham que se sentar pode se contaminar, se conversar pode se contaminar, um beijo ou abraco pode se contaminar, eu acho isso ignorância, eu tenho compaixão, sou amiga, mas acho que ela procurou porque ela quis. (E 20).

O relato que refere a culpa da contaminação ao sujeito portador apresenta uma concordância com o estudo no qual os sujeitos declararam que a irresponsabilidade nos intercursos sexuais culmina na soropositividade, o que acarreta o sentimento de culpa (CAMARGO; BERTOLO; BÁRBARA, 2009).

A análise que menciona os mitos sobre a contaminação pelo vírus aborda a necessidade de trabalhar questões voltadas para a saúde dentro da sala de aula. Faz-se necessária a existência de um profissional capacitado para as questões educacionais acerca do tema.

\section{Dimensões e complexidades obser- vadas pelos estudantes as pessoas com HIV/Aids}

Nesse item, os sujeitos relatam os preconceitos vivenciados, doenças associadas à tristeza e o não uso de preservativos nas relações conjugais. Para elucidar essa questão, serão expostos os seguintes fragmentos:

As pessoas veem como uma pessoa totalmente incapacitada de fazer qualquer coisa, não ver como uma pessoa normal, eu já sofri discriminação só por estar com ele, já perdi amigos. (E 05).

Ela não fica com mais ninguém. Ela tem marido, ele tem Aids também porque eles não se preveniam. Ela acabou ficando louca; fala coisa com coisa; ficou em depressão; ficou doente. Hoje em dia ela não faz mais nada, não pode trabalhar. (E 47). 
As falas expressam o sentimento de preconceito e discriminação contra as pessoas que vivem com HIV/Aids. Estudo semelhante de âmbito nacional realizado em 2011 identificou que, entre adultos, ainda existe a estigmatização e o despreparo associados à Aids e a temas relacionados com a sexualidade, apesar de campanhas na mídia nacional (GOMES; SILVA; OLIVEIRA, 2011). A desmistificação dos sentimentos expressados pelos estudantes requer competências e habilidades desempenhadas com segurança e conhecimento pelos componentes da equipe de saúde, com ênfase no Enfermeiro, que assiste o doente com cuidados complementares no ambiente hospitalar, na atenção básica e ambiente educacional.

Assim, as falas que expressam a impossibilidade da pessoa em exercer suas atividades normais, como trabalhar, ter relação sexual ou ter filhos, são reapresentadas como uma complexidade em relação ao adoecer pelo vírus. Tal conjetura sugere que os entrevistados não estão conscientes do tratamento da doença - a Aids é uma doença para o resto da vida, semelhante a uma doença crônica, tal como a diabetes, que requer disciplina e exige alguns cuidados, como hábitos saudáveis na rotina diária, dormir cedo, alimentar-se bem, não beber, não fumar e manter alimentação balanceada (CAMARGO; BERTOLO; BÁRBARA, 2009).

\section{Vulnerabilidade de pessoas com o HIV devido à infecção do vírus}

Nessa subclasse, observa-se a ligação entre a religiosidade e os depoimentos. Os discursos que abordam esse tema exibem uma forma de fortalecimento ao indivíduo no enfrentamento das fragilidades expostas pelo HIV, como visualizado a seguir:

Ele não se tornou uma pessoa triste, ele aceitou Jesus, realmente deve ter seus momentos de tristeza, ele é uma pessoa alegre, que luta para aproveitar o tempo que ele tem. (E 48).
Sinto compaixão, a Aids não tem cura, só tratamento eu acho que só tem Jesus porque não tem outra não. (E 03).

De acordo com os trechos, os sujeitos utilizam a divindade como refúgio; o símbolo da instituição religiosa alivia o sofrimento do portador e de familiares. A religião é uma forma de acolhimento e de apoio ao enfrentamento dos aspectos morais das repercussões clínicas do adoecimento pelo HIV. Aceitar a condição da doença pode ser parte de um processo que inclui a construção de novas identidades, como ser incluído em um grupo religioso (PAIVA ET AL., 2011). Desse modo, a religião reapresenta um fortalecimento adiante da fragilidade do adoecer.

Outro aspecto identificado nessa subclasse é que apesar da Aids ser considerada doença crônica que possibilita ao indivíduo infectado melhorar seu aspecto físico e emocional, a associação da doença ao preconceito e a ideia da morte pelo HIV é ligada às práticas sexuais desviantes que comprometem as regras sociais. Essa realidade pode ser evidenciada no trecho: "[...] ela descuidou, tinha relação sexual com um e com outro, toda noite ela saia para balada, bebia, usava drogas" (E 35).

Nesse sentido, as práticas sexuais consideradas desviantes permitem a apresentação de pensamentos negativos em relação a quem tem o vírus. $\mathrm{O}$ depoimento em relação ao uso de drogas nas falas se sobressai como um fator de extrema importância, pois aborda uma das principais vias de contaminação.

O julgamento da pessoa com HIV/Aids remete à culpa pela contaminação, ao não uso do preservativo e à multiplicidade de parceiros como causas da contaminação.

A culpa, ante o HIV/Aids, demonstra constituir uma questão intrigante por envolver aspectos relacionados com o juízo de moralidade, de consciência e de justiça. Os resultados demonstram que a contaminação do sujeito é decorrente do seu comportamento de risco estabelecido pela 
sociedade, envolvendo aspectos relacionados com o juízo de moralidade, de consciência, de justiça anteriormente prescrita pelo social. Os sujeitos entrevistados não percebem que os modos de transmissão de uma doença têm razões mais sociais do que individuais (FERREIRA; FAVORETO; GUIMARÃES, 2012).

Diante dos resultados, ressalta-se a importância da promoção de programas educativos em parceria com os profissionais de saúde nas instituições de ensino, em especial ao profissional enfermeiro, que contribuam para a conscientização dos estudantes quanto à importância da prevenção e promoção em saúde no ambiente escolar, como também a desmistificação em torno do HIV/ Aids. Assim, menos estigmas serão formulados nessa relação, e aquele que portar o HIV/Aids poderá ter uma melhor qualidade de vida ao conviver com pessoas menos preconceituosas.

\section{Considerações finais}

Este trabalho fortalece as discussões de jovens e adultos acerca da pessoa que vive com HIV/Aids. Por meio deste estudo, foi possível identificar diversos conteúdos que compõem a representação social da Aids para os escolares que estão inseridos na EJA e sua influência acerca dos indivíduos soropositivos, apresentando-se como uma realidade vivida. A representação da doença abarca diferentes aspectos, com destaque para a vulnerabilidade do portador do vírus, os medos, sentimentos e vivências acerca da doença. Os sujeitos reapresentam o preservativo e a multiplicidade de parceiros como principal motivo para a contaminação pelo vírus.

Não se pode ignorar que a Aids vem se construindo ao longo dos anos como uma doença do medo. Sabe-se que é difícil mudar opiniões preconceituosas, conceitos e estigmas perante a história da Aids, por isso faz-se necessário alertar as instituições de ensino quanto ao papel educacional no que tange à educação social para a formação de pessoas com visões abrangentes aos estigmas da doença e menos preconceituosas.

Percebeu-se que a promoção à saúde sexual dos estudantes necessita integrar temas sobre a conscientização social e educação e saúde: é a partir daí que os profissionais de saúde devem colaborar para a desmistificação de sentimentos e atitudes referentes ao portador do HIV/Aids. Assim, reforça-se a necessidade do Enfermeiro em promover novas estratégias de ensino para abordar esse conteúdo, na concretização de uma educação transformadora, que contribuam para mudanças sociais rumo a uma sociedade justa.

Nesse caminhar, no que se refere à saúde, o tema em questão traz implicações importantes para o campo da enfermagem, para as práticas de promoção da saúde, especificamente de prevenção da disseminação do vírus e da doença. 


\section{Referências}

BRASIL. Ministério da Saúde. Secretaria de Atenção à Saúde. Saúde na Escola. Brasília, DF: Ministério da Saúde, 2009.

Ministério da Saúde. Secretaria de Vigilância em Saúde do Departamento de DST, Aids e Hepatites virais. Boletim Epidemiológico. Brasília, DF: Ministério da Saúde, 2015.

CAI, Y. et al. Study of HIV/Aids-related knowledge among junior high-school students in Shanghai, China. Int J STD AIDS, Londres, v. 23, n. 3, p. 9-12, 2012.

CAMARGO, E. A. I.; FERRARI, R. A. P. Adolescentes: conhecimentos sobre sexualidade antes e após a participação em oficinas de prevenção. Ciênc. Saúde Coletiva, Rio de Janeiro, v. 14, n. 3, p. 937-946, 2009.

CAMARGO, V. B.; BERTOLDO, R. B.; BARBARÁ, A. Representações sociais da Aids e alteridade. Estud. pesqui. psicol., Rio de Janeiro, v. 9, n. 3, 2009.

CHAVES, A. C. P. et al. Conhecimentos e atitudes de adolescentes de uma escola pública sobre a transmissão sexual do HIV. Rev Bras Enferm, Brasília, DF, v. 67, n. 1, p. 48-53, 2014.

DANTAS, M. S. et al. HIV/Aids: significados atribuídos por homens trabalhadores da saúde. Esc Anna Nery, Rio de Janeiro, v. 19, n. 2, p. 323-330, 2015.

FERREIRA, D. F.; FAVORETO, C. A. O.; GUIMARÃES, M. B. L. A influência da religiosidade no conviver com o HIV. Interface (Botucatu), Botucatu, v. 16, n. 41, p. 383-393, 2012.

FERREIRA, I. D. R. C. et al. Percepções de gestores locais sobre a intersetorialidade no Programa Saúde na Escola. Revista Brasileira de Educação, Rio de Janeiro, v. 19, n. 56, p. 61-76, 2014.

GOMES, A. M. T.; SILVA, E. M. P.; OLIVEIRA, D. C. Representações sociais da Aids para pessoas que vivem com HIV e suas interfaces cotidianas. Rev. Latino-Am. Enfermagem, Ribeirão Preto, v. 19, n. 3, p. 8, 2011.

JODELET, D. As representações sociais: um domínio em expansão. Rio de Janeiro: UERJ, 2001.

NAGAMATSU, M. et al. HIV prevention through extended education encompassing students, parents, and teachers in Japan. Environ Health Prev Med, Nova York, v. 16 , n. 6 , p. $350-362,2011$.

NASCIMENTO, A. R. A.; MENANDRO, P. R. M. Análise lexical e análise de conteúdo: uma proposta de utilização conjugada. Estud. pesqui. psicol., Rio de Janeiro, v. 6, n. 2, p. 72-88, 2006.

OLIVEIRA, D. C.; GOMES, A. M. T.; MARQUES, S. C. Análise estatística de dados textuais na pesquisa das representações sociais: alguns princípios e uma aplicação ao campo da saúde. São Paulo: Casa do Psicólogo, 2005. p. $157-200$

PAIVA, V. et al. A sexualidade de adolescentes vivendo com HIV: direitos e desafios para o cuidado. Ciênc. Saúde Coletiva, Rio de Janeiro, v. 16, n. 10, 2011.

PERUCCHI, J. et al. Psicologia e políticas públicas em HIV/Aids: algumas reflexões. Psicologia \&t Sociedade, Belo Horizonte, v. 23, n. esp., p. 72-80, 2011.

PIAU, F. S.; PEREIRA, B. B. Orientação sexual na educação de jovens e adultos: os alunos como sujeitos ativos na construção de conhecimentos sobre as doenças sexualmente transmissíveis. Cadernos da FUCAMP, Monte Carmelo, v. 11, n. 14, p. 93-103, 2012.

SANTOS, É. I. et al. Revisão integrativa de literatura acerca da atuação do enfermeiro na prevenção da transmissão de HIV entre idosos. Revista Augustus, Rio de Janeiro, v. 17, n. 34, p. 18-31, 2012.

SERRA, A. et al. Percepção de vida dos idosos portadores do HIV/Aids atendidos em centro de referência estadual. Saúde em Debate, Rio de Janeiro, v. 37, n. 97, p. 294-304, 2013.

Recebido para publicação em maio de 2016

Versão final em outubro de 2016

Conflito de interesses: inexistente

Financiamento: não houve 